\title{
CONGREXPO: LA ENORMIDAD COMO PROGRAMA IDEOLÓGICO
}

\section{CONGREXPO: BIGNESS AS AN IDEOLOGICAL AGENDA}

lgnacio Senra Fernández-Miranda

RESUMEN El anallisis del primer edificio de gran escala llevado a cabo por OMA, el Congrexpo de Lille, sirve para comprender

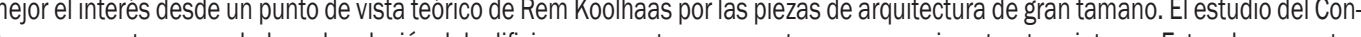
grexpo se centra por un lado en la relación del edificicic con su entorno y por otro, en su propia estructura interna. Estos dos aspectos posiciones teóricas anteriormente encontradas: Unidad o fragmentación, universalidad o especificidad, Zeitgeist o Genius loci la atención a estas oposiciones características del conflicto entre el Movimiento Moderno y la primera postmodernidad, definen un programa ideológico moderno, aunque a la vez critico sin ser reaccionario. Este programa se desvela a través de la profundización en el análisis del Congrexpo y del master plan del que deriva

PALABRAS CLAVE OMA; Rem Koolhaas; Lille; Bigness; Zeitgeist; Genius loc

SUMMARY The analysis of Congrexpo at Lille, first large-scale building by OMA, is carried out in order of a better understanding of Koolhaas theoretical interest in large-scale architecture. The study is focused on both building-context and building-structure relationships. This two aspects show how irony in Koolhaas goes beyond simple provocation, understood as the logic capable of fitting together theoretical stances which were considered as contradictories before: unity vs. fragmentation, universality vs. specificity, Zeitgeist vs. Genius loci. The attention to these oppositions characteristics of the confict between Modern Movement and first
postmodernity defines an ideological agenda which is modern and simultaneously critical with modern principles but without being reactionary. This arenda is revealed throush deeper analysis of the Congrexpo and the maste-plan from which it derives. PALABRAS CLAVE OMA; Rem Koolhaas; Lille; Bigness; Zeitgeist; Genius loci

Persona de contacto / corresponding author: isenra@yahoo.es. Escuela Técnica Superior de Arquitectura. Univerisdad Politécnica de Madrid
E n 1989, como resultado de un concurso, Rem Koolhaas recibió el encargo de dirigir y coordnar el proyecto de planificación urbana de mayor escala que en aquel momonto se llevaria a cabo en eupán el desanolo de la zona que ocuparia la nueva ercón

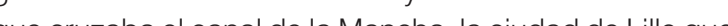
daba una posicín pivilejada sitúndose a menos le hora y media en tren de Londres, Pals y Busels.

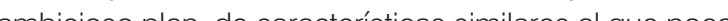
años despús se leaŕa a cabo para aspiraba a modificar completamente el destino de la citdad, creando un nuevo centro de actividad en tomo a la nueva estación. Pocos pod'an imaginar que un encargo de semejante envergadura recarí sobre el joven arquecto holandés todav́a más ocupado en teoŕa y polemicas que en la construccín de tan complejes programas. Pero la ambicín det alcalde de Lile, Piere Mauroy encajaba a a perfeccín con la vangurerdsta visión de OMA de la metrópolis: congestión, superposición de funciones e infraestructuras, programas culturates y comerciales como base para el desarrollo on altura. El nuevo master plan de Lille constitúa una Koolhaas de poner en práctica lo aprendido anteriormen-

\section{AARQUITECTURA COMO ALTERNATIVA AL}

El escepticismo de Koolhaas con el urbanismo le hace conflar direclamente a la arquitectura la solución de cas 120 hectáreas de terreno vacio, un espacio residual juncruce de una autopista urbana sobre lo necuencia de vís de a antigursta ún de sencillo y contundente plan de compleja ejecución tres piezas de escala gigntesca senvín para resolver todo e conjunto, en total más de $800000 \mathrm{~m}^{2}$ de programa el Forum Triangular encargado a Jean Nouvell la nueva estacín de TGV sobre cuya abjeta longidina se posaín siete torres realizadas por diferentes arquitectos, finalmente el Grand Palais o Congrexpo, la pieza para celebración de grandes eventos de la que se hara cargo

Planeamiento consiste en la creación de oportunidades, más que en el orden y el control del territorio La arquitectura si es suficientemente grande puede llegar a adoptar funciones anteriormente confiadas al urbanismo y puede por tanto ser abordada con herramientas tradicionalmente ligadas al planeamiento Las piezas de arquitectura de gran escala son urbanas en sí mismas independientemente de la ciudad Su autonomía unida a su propia condición urbana las convierte 
1. Lille, plan general de ordenación: 1. Estación TGV; que; 5. Saint-Mauricic; 6 . Congrexpo.

2. Malévich, Suprematismo (Supremus n58) 1916, óleo sobre tela, 79,5570,5. Museo Ruso, Leningrado. 3. O.M. Ungers, R. Koolhasas, H. Kollhoff, A. Ovaska,
P. Riemann, cities within the city, Berlin 1977.

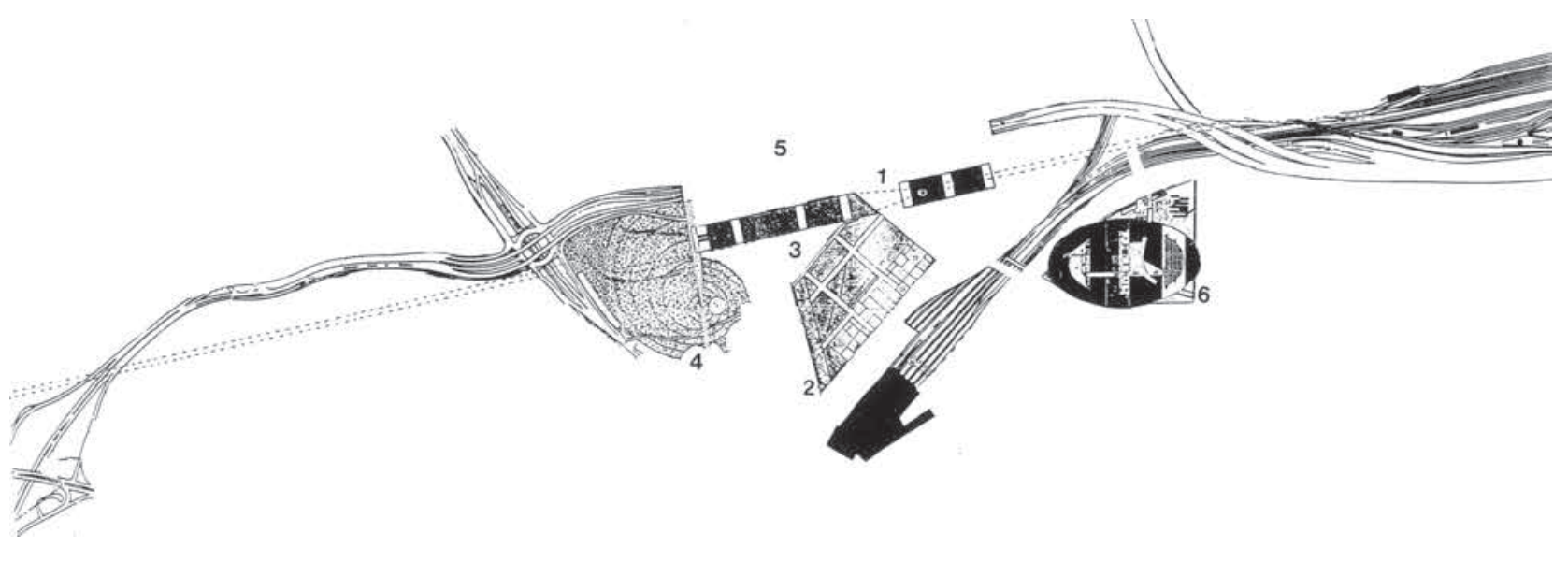

en catalizadores capaces de modificar la ciudad por si solas, sin necesidad del planeamiento entendido como disciplina de regulación normativa. Koolhaas afirma:

El planeamiento ya no tiene sentido, lo hemos saboleado y ridiculizado hasta el punto de cerrar departamentos enteros en las Universidades... Todavía culpamos a otros por una situación de la que solamente nuestro incurable utopismo y desprecio son responsables... Una profesión que insiste en sus fantasías, su ideología, su pre tensión, su llusion de implicación y control, y que por eso es incapaz de concebir nuevas modestias, intervenciones parciales, reajustes estratégicos, posiciones comprometidas que puedan influenciar, redinigir y triuntar en términos más limitados, reagrupar, empezar de cero incluso, pero

La aceplación de la ciudad existente, "más que nunca, la cludad es lo que lenemos", y el entendimiento de urbanismo comouna estralegla de acupuntura a base de piezas arquitectónicas, se traduce en una solución donde losvacios entrelas tres plezas, dejande ser espacios restaules para convertise en oponundades para la cludad, plazas, espacios verdes o polon as composicion as recios entre tas tres figus abstractas, el oval,

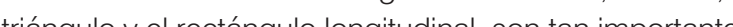
o más que las piezas ensí (iguras 1 1 2) El compleirsmo programa se consigue limitar a estas tres piezas exclusipante y todos los demás elementos de menor escala as diferentes tores de oficins los hoteles se superponen sobre ellas El sobrecoste que supone suspender las torres sobre la cubierta de la estación (entre un 8 y un 10\%) en ser capaz de tener en mente dos ideas opuestas al

se rentabiliza en forma de simbolismo, como sugiere pra una de ellas. De manera similar a lo propuesto en proyecto teórico para Berlín realizado en 1977 con O. M. Ungers, Clites within the city' (figura 3), Koolhaas confia a la inserción estratégica de las tres piezas toda una serie de transtomaciones urbanas. la conversión de una autopista en bulevar, el entendimiento de la nueva estación como puente en lagar de como fractura, ol a aparición de la plaza Fraçois Mitterrana. Se establecen así una serie de relaciones entre piezas, intersectiones, dependencias y obligaciones muluas, que hacen imposible entender las piezas como proyectos independientes. Como si se tratara de una composicion supremalsta, la culdada posicion (a) constituyen el plan.

GRAN ESCALA: ORIGEN DE CONTRADICCIONES

Se ha escrito mucho sobre la paradoja, la contradicción y la ironía en el trabajo de Koolhaas. No es nada nuevo tampoco señalar el interés de Koolhaas por el tamaño desmesurado en arquitectura. ¿Pero hasta qué punto eslán relacionados los conceptos de gran escala y contradicción en Arquitectura? Por un lado Koolhaas sostien que "solamente la Enormidad impulsa el régimen de la compleijdad que moviliza plenamente la inteligencia de la arquitectura"4. Por otro lado, ya en Delirious New York, Koolhaas se sivió de las palabras de Scott Fitgerald para definir lo que para él constituía una inteligencia de primer orden: aquella cuya prueba fundamental "consiste

1. Koolhaas, Rem: “Whatever happened to Urbanism”. En OMA; Koolhaas, Rem; Mau, Bruce: SMLXL. Nueva York: The Monacelli Press, 1995. p. 969. 2. Koolhaas ve el urbanismo no como disciplina sino como forma de pensar. La aceptación de lo existente, una idea de la ciudad compartida con Ungers Koolhaas, Rem: "Whatever happened to Urbanism", op. cit. p. 971.

3. Ungers, O.M.; Koolhaas, Rem; Riemann, Peter, Kolhoff, Hans; Ovaska, Arthur. "Cities within the City, Proposals by the Sommer Akademie for Berlin". En Lotus intermacional n.19, 1978. pp. 82-97. En esta estrategia urbana para Berlin se propone un archipiélago de microciudades morfológicamente difierentes.

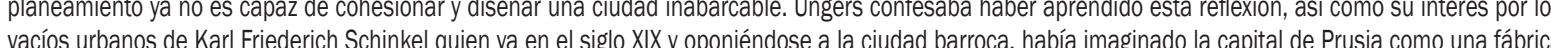
urbana salpicada de intervenciones arquitectónicas puntuales de carácter singular. Para profundizar en las resonancias entre la obra temprana de Koolhaas la de Ungers ver: Schriver, Lara: "OMA as a tribute to OMU: exploring resonances in the work of Koolhaas and Ungers". En The Journal of Architecture, vol.13. No 3. 2008. pp. 235-261; y Neumeyer, Frtzz: "OMA 's Berrin: The polemic island in the city"."En Assamblage n.11, Abril 1990. pp. 36-53.

4. La palabra Enormicad se utiliza para traducir el termino "Bigness" inventado por Koolhaas como sustantivacion del adjetivo Big: grande, termino utilizado para referirise a la arquitectura de gran escala. Koolhaas, Rem: "Bigness or the problem of large". En OMA; Koolhaas, Rem; Mau, Bruce: SMLXL. Nueva Yorr 
mismo tiempo, y mantener sin embargo la capacidad de funcionar"s. Poniendo en común ambas observaciones tenemos que para Koolhaas los proyectos de arquitectua de gran escala requieren especialmente un cierto grado de ironía y de ambigüedad, o lo que es lo mismo: el ser capaces de funcionar simultáneamente atendiendo a

Tanto la ambigüedad como la paradoja resultan conceptos enormemente difusos y genéricos si no se determina su funcionamiento concreto en arquitectura. Algunos de los arquitectos más incisivos en la crítica del Movimiento Moderno han utilizado términos como Conciliación de Contrarios, Aldo Van Eyck; Coincidentia Oppositorum, Oswald Mathias Ungers; o simplemente Contradicción. Robert Venturi, para defender desde la teoría una arquitectura no simplificadora. Pero su puesta en práctica en arquitectura dista mucho en unos y otros casos. La frase "Io uno y 10 otro en lugar de o lo uno o lo otro"6, con la que Robert Venturi invitaba a los Ilamados Arquitectos Inclusivistas a reaccionar contra la simplificación moderna, expresa de manera especialmente elocuente la actitud de Koolhaas en este sentido, pero al mismo tiempo resulta enormemente genérica. A menudo la aceptación de la contradicción se ha traducido de manera demasiado inmediata en juegos formales y compositivos, sin entrar prácticamente en cuestiones ideologicas y estratégicas. Pero no es este el caso de Koolhaas que encuentra en la arquitectura de grandes dimensiones a inevitable complejidad que le permite relacionar directamente el tamaño de un edificio con su programa ideológico, "independiente de los deseos de su arquitecto"t. En tras palabras, el mero hecho de que un edificio sea lo

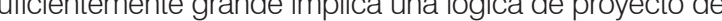
buta ben coexistir sin excluirse nim moderarse. Airgual que ocuritia I Congrexpo de Lille las contradicciones se comprometen

especialmente con algunos de los conflictos que surgieron entre arquitectura moderna y la reacción anti-moderna de males de los sesenta: la relación del edificio con el lugar la relación entre interiory exterior la relación entre coniunto y sus partes El Congrexpo consitituye la materialización de dicho programa ideológico.

DESLOCALIZACIÓN VS CONTEXTUALISMO

Una de los principales reproches en contra de la arquitectura moderna ha sido el de su despreocupación por contexto físico donde un edificio se asienta, el lugar. Los principios de industrialización y universalidad modernos acabaron generando una reacción contextualista contra la que Koolhaas se ha pronunciado en no pocas ocasiones ${ }^{8}$. A pesar de que la teoría oficial de OMA presenta el Congrexpo como una intervención autónoma y ajena al lugar que podría estar en ese o en cualquier otro sitio, puede apreciarse al mismo tiempo un esfuerzo importante por relacionar el nuevo edificicio con su entorno físico concreto. A primera vista, el enorme huevo parece posarse sobre el terreno como lo haría un ovni aterrizando junto a las vías del tren, ajeno a toda consideración sobre el lugar. E propio Koolhaas se ha esforzado en desvincular del territorio el proyecto de Lille al señalar su imprecisa dirección situándolo no en un contexto geofísico sino en relación a tiempo que lo separa de otras ciudades (a 40 minutos de París, a 70 minutos de Londres, a 18 minutos de Bruseas). Un nudo en la red de transportes que, como el aeropuerto internacional de Atlanta, podria estar en cualquier otro lugar. La abstracción por medio del tiempo sirve a una clara intención de deslocalización en terminos geográficos concretos, de enajenación de los objetos sensibles que componen el lugar. La abstracción de la forma ovoide sugiere esta misma idea a primera vista, además de poseer un significado simbolico previo a cualquier contemplación del terreno. No es la primera vez que el

5. F. Scott Fitzgerald “The Crack Up”, citado en Koolhaas, Rem: Delirious New York: A Retroactive Manifesto for Manhattan. Nueva York: The Monacelli Press,

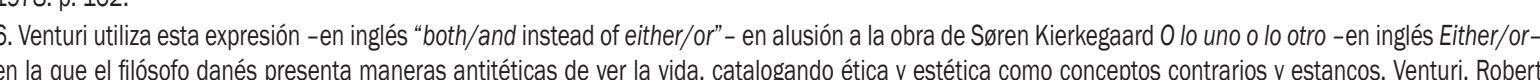
.

7. Hoonhas, Rem: "Bigness or the problem of large", op. cit. p.496.

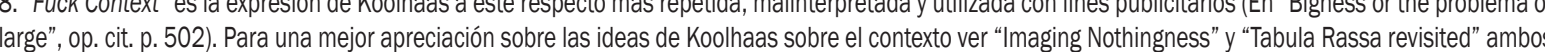
large", op. cit. p. 502). Para una mejor apreciación sobbr las ideas de Koolhaas sobre el contexto ver "Imaging Nothingness" y "Tabula Rassa revisited" ambos huevo aparece en la obra de Koolhaas. Desde su primer manifiesto, en 1975, donde se anunciaba el nacimiento de OMA con un elocuente dibujo de una torre rompiendo el cascarón al salir de un huevo, esta forma heredada de a iconografía surrealista de Dalí se ha convertido en un símbolo de identidad de la firma, una marcag (igura 4) Sin embargo la forma ovoide de Lille se ha tratado de explicar por razones matemáticas indicando que la forma resultante consigue encerrar el máximo volumen interior con el mínimo contacto con el exterior. Pero de ser esta la razón principal habría resultado un círculo una forma cuya máxima abstracción geométrica encaja mejor en el discurso de la indiferencia al lugar del que se hace gala La cuestión es si las razones por las que el trazado de edificio fue definitivamente un huevo y no un círculo o una elipse, forma que sí aparece al inicio del proceso, responden solamente a cuestiones simbólicas, o también a las características específicas del lugar.

Lejos de superponerse indiscriminadamente al tejido urbano existente, como caído del cielo, algo que sí ocurría en los primeros croquis y maquetas del master plan (figura 5) y en otros trabajos de OMA como Exodus o la casa Spear en Miami, la solución final para el Congrexpo parece encajar de forma muy ajustada en el vacío que queda entre las vías de tren y la autopista. El extremo norte del edificio se estrecha convirtiendo la elipse original en un huevo que encaja más ajustadamente en el encuentro entre las vías y la autopista. De hecho el huevo no se posa directamente sobre el terreno; un podio de forma más o menos triangular que coincide exactamente con a extrusión del solar sirve como base para su aterrizaje. Tal como ocurria en la propuesta teorica para Manhattan The city of the captive Globe, 1972, donde las manzanas extruidas de mârmo brillante servian como base para una extensa colección de peculiares objetos arquitectónicos, completamente autónomos y ajenos a la relicula sobre la que se asentaban, el Congrexpo de Lille retoma esa doble condición de autonomia y dependencia simultáneas del edificio con respecto al sistema urbano.
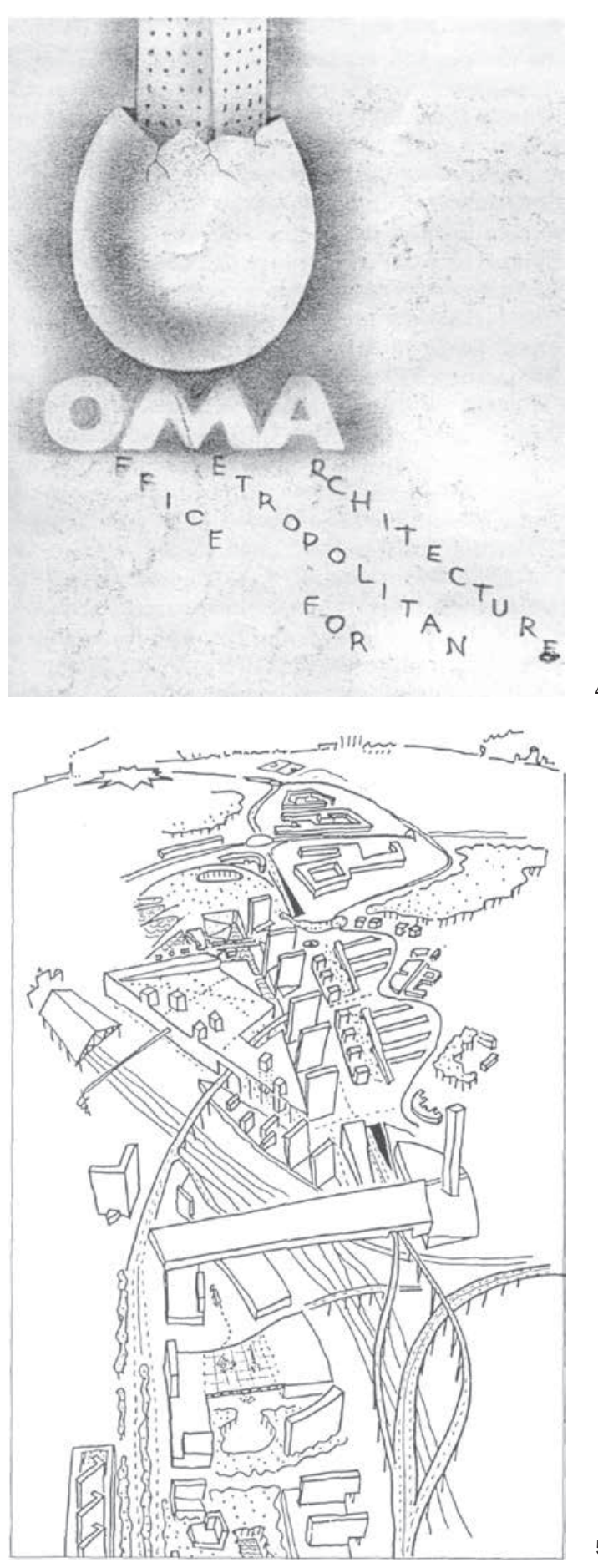

9. Utilizado en innumerables ocasiones por Dalí como símbolo de retorno a la matriz, a la vida intrauterina, Koolhaas I o ha utilizado desde el anuncio del nack miento de OMA con carácter más o menos simbólico. El huevo de Columbus Center, la central martíma de Zeebrus gese

de Lille utilizan esta geometría en planta, en sección o en tres dimensiones, conviritiéndolo en seña de identidad.

10. En los primeros croquis para el master plan la pieza de exposiciones y congresos de la que se encargaría OMA es un rectángulo alargado superpues Archizoom. 
6. Congrexpo, Croquis mostrando cómo el tráicico roen el edificio.

7. Maqueta de trabajo del Congrexpo cuando las auopistas aún lo atravesaban.

8. Terminal martima de Zeebrugee. Autopistas y pasarelas como sistemas de anclaje.

9. O. M. Ungers. Propuesta para Tiergarten Viertel,

Berlín 1973. ¿Genius loci o Zeitgeist?
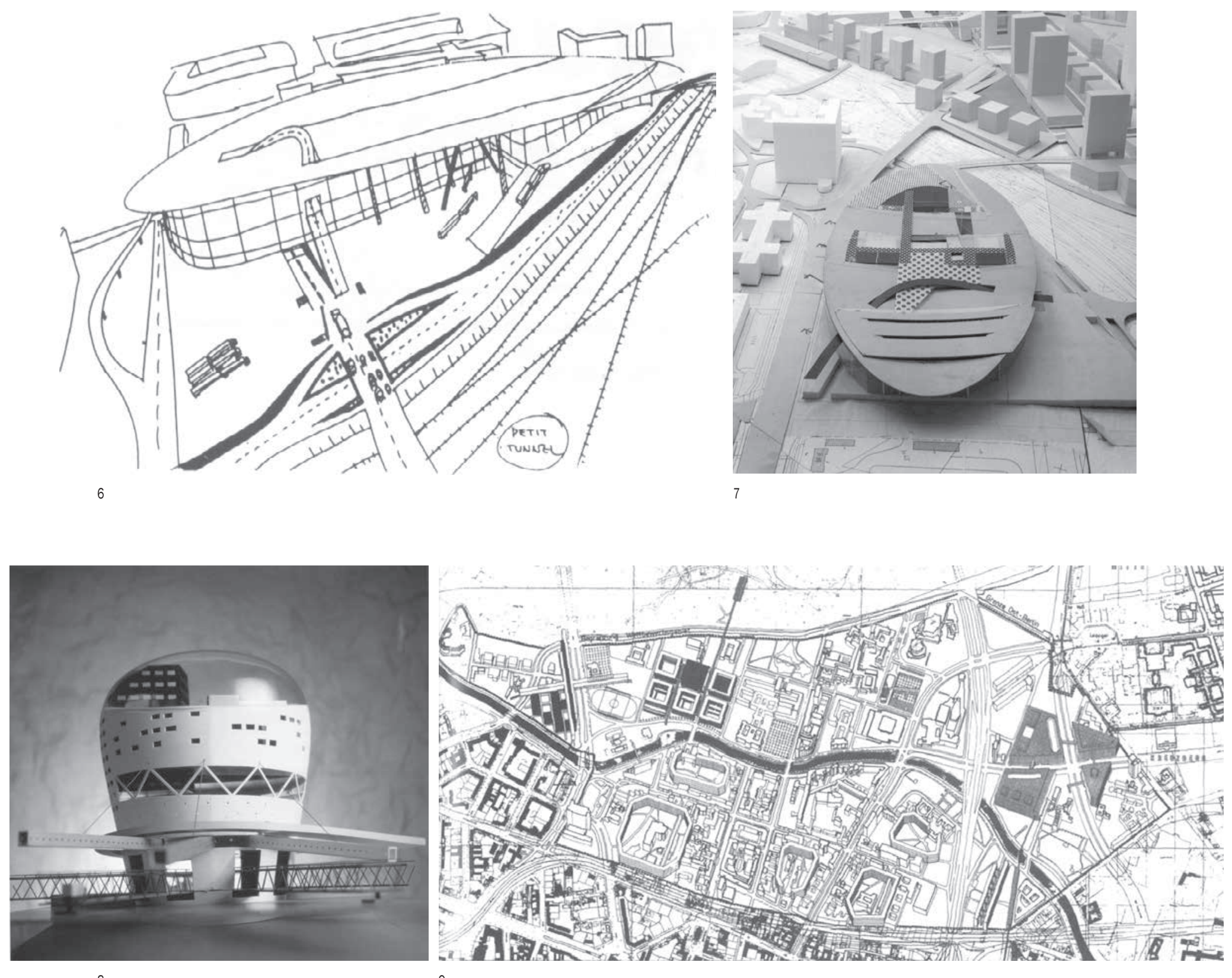

La obsesiva abstracción geométrica de los proyectos de OMA no está en absoluto reñida con la contextualización del proyecto, o más precisamente, con la concreción de la idea en relación a la especificidad de la realidad Otros proyectos de esta misma época como el triangulo presentado en 1988 al concurso para el Insfituto Holandés de arquitectura en Rotterdam, o el proyecto de hotel y palacio de congresos en Agadir 1990 se centran también en esa relación entre universalidad y particularidad, entre abstracción y materialización'11. En palabras de Jean-Paul Baietto, máximo responsable del consorcio público-privado creado para llevar a cabo el proyecto de Lille: "Al principio del siglo XX se podía ser un héroe proponiendo generalidades: pero a final de siglo solamente lo hiper-especifico resulta creible"12 Koolhaas es plenamente consciente de ello, pero valora tanto como la súper-especificidad, la universalidad moderna de sus deas, hasta el punto de llegar a catalogarlas en un libro de "patentes universales de modernización"

Si hoy sobrevolamos el Grand Palais de Lille, resulta difícil determinar si el edificio de Exposiciones y Congresos se ha adaptado al trazado de la autopista y de las vías de tren, o si por el contrario son éstas las que deben su trayectoria al gigantesco edificio ovoide. ¿Que fue antes el huevo o las infraestructuras de transporte? Los contactos tangenciales de la pieza ovoide tanto con la autopista como con las vías ferroviarias tienen la clara intención de acentuar aún más si cabe el tamaño del edificio. Se trata de una declaración de intenciones. Un edificio con esa geometría parece necesitar cierto margen a su alrededor, un espacio de respeto, pero al contrario de lo que su geometría sugiere no puede rodearse. Nadie se podía imaginar al ver la maqueta que ese objeto encajaría de manera tan ajustada en un límite previamente existene. No hay margen, el edificio se adapta con precisión

milimétrica a las curvas descritas por vías y autopista traen al arquitecto holandés. En dicho cruce de infraestructuras se sit́r el vérice de la paŕbola que conforma el huevo, que se eleva del suelo generando en la parte superior el graderí de la sala de conciertos y en binferio un acceso tanto peatonal al Zénith, como de vehículos aparcamiento ¿ No son esto mecanismos de vinculación entre el obieto y el contexto físico que lo rodea?

Además en los primeros croquis y maquetas del proyecto se puede observar cómo un brazo de autopista se eleva y atraviesa la fachada y la cubierta para, tras describir una cunva volver a introducirse en el edificio y salir por la fachada Un gesto de anclaje a modo de puntada de costura que intenta mantener el edificio unido al terreno y evitar asi que salga volando de nuevo. (figuras 6 y 7 ) Son estas lineas de transporte, tanto vías férreas como autopista, las que sirven a Koolhaas para establecer la unión entre el edificio y el lugar. Se trata de una estrategia para contextualizar la intervención a través de las infraesructuras existentes, utilizada ya en proyectos anteriores de OMA como el Kunsthalle de Rotterdam, 1987, donde la via de servicio de la autopista atraviesa el edificio, mas evidentemente en la estación marítima de Zeebrugge $\mathrm{e}^{14}$, 1989, donde los puentes de acceso rodado y las pasarelas peatonales sirven para anclar literalmente la terminal aerostática (figura 8). Una estrategia en deuda una vez más con la visión de Ungers de la ciudad. Fue ê quien, en propuestas como la de Tiergarten Viertel, Berlin 973 (figura 9), se preocupó por el contexto de manera realista, centrandose en una aceptación de lo existente compatible con la abstracción y la autonomía de la nueva aquectura, mostrando asi su oposición a la melancolía Vencististas y regionalistas. En Tiergarten

11. El proyecto de Aggadir representa mejor que ningún otro esa doble condición universal-particular. La propuesta de planta cuadrada propone un nuevo tipo un edificio que se torna autónomo y que podría llegara a exportarse a cualowien otro lugia Monacelli Press, 1995. p.1208.

13. OMA-AMO; Koolhaas, Rem: Content, Köln: Taschen, 2004.

14. Para Alfonso del Pozo es precisamente la central de Zeebrugee, 1989, la que abrió para OMA la senda de los edificios de gran escalal vinculados a grandes Infraestructuras. Este intercambiador es la mejor muestra del enorme interés por las infrestucturas en la obra temprana de OMA. Pozo y Barajas, Alfonso del Construcción, 2009. pp. 165-169. 

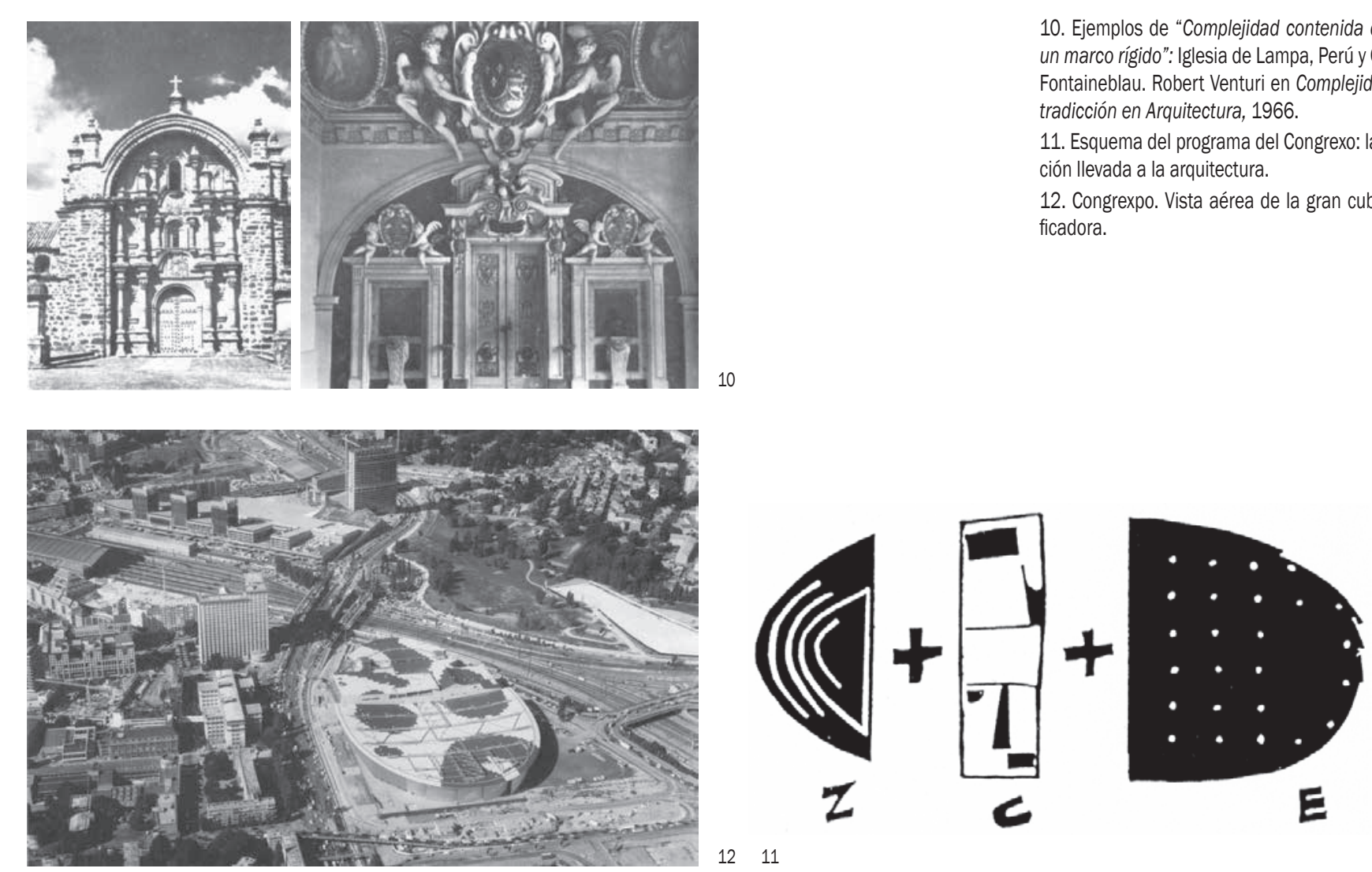

situaciones de la ciudad existente, pero "especialmente a aquellas sin pedigri historico como intersecciones de tráfico, vertederos, o bloques cerrados incompletos"15, algo que también ocurre en el proyecto de Koolhaas para Lille.

UNIDAD VS FRAGMENTACIÓN

Otra de las grandes denuncias contra la arquitectura moderna fue la obsesión por lo que Venturi definió como: la fácil unicad conseguida mediante la exclusion, frente a la difícil unidad conseguida a través de la inclusión"10. Algo similar apunta Koolhaas cuando se refiere a la gran escala: "la imposibilidad de controlar tal tamaño mediante un solo gesto arquitectónico, provoca la autonomía de las partes", pero puntualiza que esto no es lo mismo que la fragmentación, ya que "las partes permanecen comprometidas con el conjunto"

En la estructura del Congrexpo la dualidad entre unidad de conjunto y autonomia de las partes es evidente. Se podira decir que este estáconstituido portes edilicios
10. Ejemplos de "Compleijidad contenida dentro de Fontaineblau. Robert Venturi ien Compleijidad y Contradicción en Arquitectura, 1966. 11. Esquema del programa del Congrexo: la zonificación llevada a la arquitectura. 12. Congrexpo. Vista aérea de la gran cubierta unia de la gran cáscara ovoide, parte la estructura en dos de unión entre las otras dos partes para construir la gran apuntado por Koolhaas con respecto al edificio de gran escala y su efecto sobre la ciudad. 'Siempre he estado que estos implican en batificilidad en a fagmentación que producan, en la modo en que su propirmentatud se convierte en antídoto contra esta fragropla magniEl mecanismo utilizado cen la configuración interior de congrexpo coincide con la "contención de comperioncon mexpo cle un maco rido" explicada por Robert Ven-

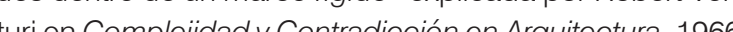
A través de jemplos escogidos de foma indisciminada, villa Saboya fachadas de iglesias barrocas y ciudades muralladas (figur 10), Venturi presentaba y cidea de forma clara y aseguraba "La compleiddad contenida podra ser uno de los métodos viables para manejar el caos ur bano a través de un uso creativo de la zonificación"1". ¿A caso no es esto lo que OMA propone en el interior del Congrexpo? (figura 11): la zonificación como estrategia capaz de crear diferentes posibilidades sin llegar a fijar configuraciones. Si en el caso del master plan la arquitectura se presentaba como alternativa al planeamiento ahora son las herramientas propias del urbanismo la que se plantean como alternativa a la arquitectura.

Esta zonificación apenas es perceptible en una vis aérea. A la clara geometría unificadora hay que sumar el patrón de manchas pixeladas superpuesto a las tres cubiertas que sirve para camuflar los límites entre ellas (figura 12). Sin embargo se manifiesta claramente al exterio a través de diferentes fachadas. A pesar de afirmar que "la aspiración humanista de honestidad está condenada y que la arquitectura de interior y exterior pasan a ser proyectos independientes"20, Koolhaas decide ser honesto en este caso y mostrar las tres partes autónomas como volúmenes independientes adosados. Los materiales utien diferentes alturas y la fragmentación del plano de faLa continuidad de la línea ovoide en planta resulta ser

18. Koolhass Pen. "Encontrando Libertades: Conversaciones con Alejandro 7 19. Venturi, Robert: Complexity and Contradicction in inchnitecture, op. cit. p. 72. 20. El problema de discrepancia entre interior y exterior en los edificios ya fue tratado en Delirious New York al introduciri el concepto de lobotomía. Anteriormen-

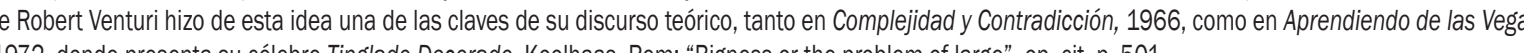
celachada. La do el perímetro del zócalo en lugares donde la cubietta desaparece. (1) por tres sistemas estructurales independientes. es erímetro sin embargo el esbelto orden de pilares que sujeta la cubierta se manifiesta unitario, queriendo perrumpido por el edificio de congresos. Las columnas del espacio de exposiciones son en su maporía interiores, pero on el frente de acceso, justo antes del encuentro con la pieza de congresos, el cerramiento se dobla hacia el interior dejando las columnas perimetrales al exterior. Al otro lado del edificio de congresos, como si el Zenith y el espacio de exposiciones compartieran un solo sistema estructural, las esbeltas columnas aparecen de nuevo, sujetando esta vez unas cerchas que quedan lla de hormigón que soporta las escaleras de evacuación

Como si de una instalación de Christo y Jean Claude se cuantitativos a cerca de su construcción: cantidad de suelo excavado, de hormigón vertido, de acero lamina-
do, Iongitud de conductos de aire presentados siempre en comparación con otros números llamativos, los de estructuras como la pirámide de Ghiza, distancias entre ciudades... El texto sirve para dar una idea de la inmensidad del objeto y del esfuerzo que ha supuesto lizados en las diferentes partes, la línea de cornisa rota chada revelan la verdadera estructura interior del huevo. po de Lille como una interminable colección de datos abstracción: elhuevo. La paradoja surge de nuevo, la pie- 


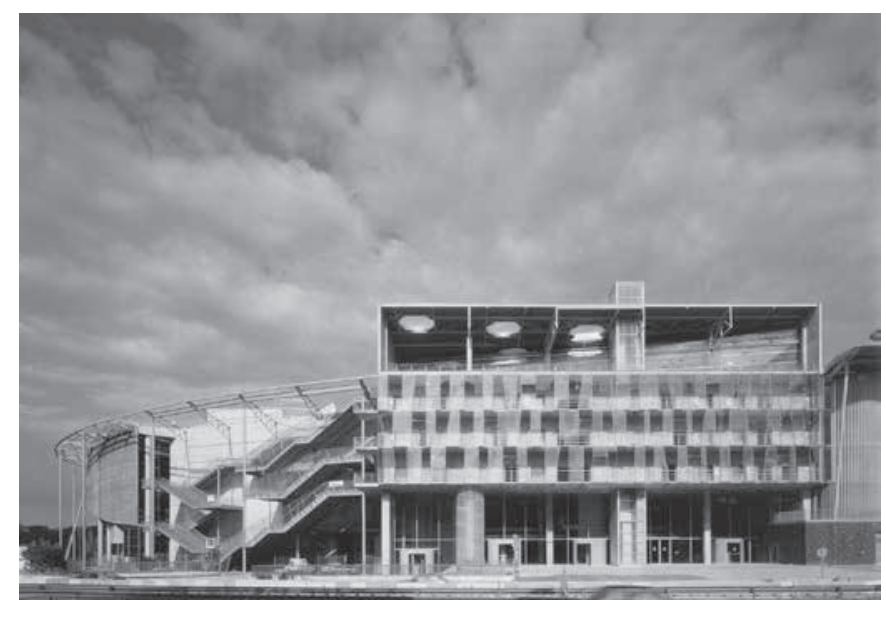

bién en el caso de las obras de Christo, para justificarse como objetivo mismo, como reto a alcanzar. El logro de haber sido llevado a cabo es más que suficiente para justificar su éxito. Así lo declara el propio Koolhaas al comparar el porqué de la gran escala para el arquitecto con el porqué del Monte Everst para el alpinistración material pero en este caso también ideob́óc La arquitectura de OMA ha sido relacionada con la Láa y con la ambigüedad demasiadas veces, muchas de ellas a ludiendo a simples oposiciones epidérmiches interiores lujosos y exteriores puritanos, detalles muy cuidados frente a a ausencia total del detalle. Otras veces ce ha hecho de forma genérica aludiendo a una arquitectura surrealista pero con sentido común visionaria pero realista, iberadora pero opresora . No se pretende pues volver a caer en estas generalidades que poco profundizan en la verdadera compleijdad del pensamiento Koolhaas. Como ya denunciara Richard Ingersoll, no es la ironía por sí misma la que aporta valor a la arquitectura de Koolhaas. Es más, Ingersoll señala que "su uso va en detrimento del valor arquitectónico y ofrece una base lamentable para el planeamiento" Es su uso como instrumento para compatibilizar posiciones antitéticas lo que la convierte en una herramienta de valor en manos

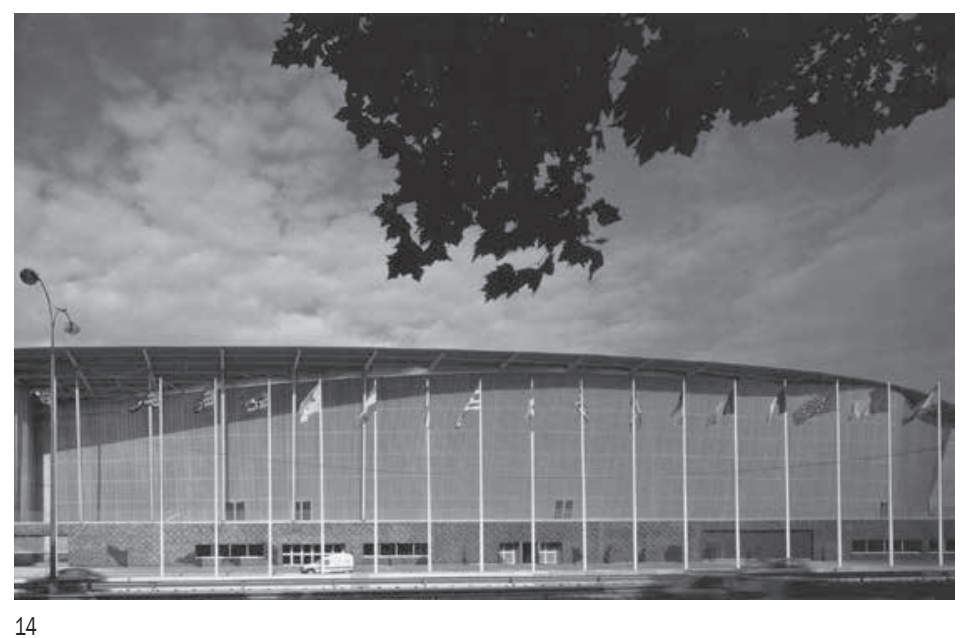

de Koolhaas. Dicho de otra forma: "Su verdadero valor es denunciar algunas de las tendencias represivas del MoviEl programa idónico un entoque reaccionario El programa ideológico de OMA encarnado en et Congrexpo de Lille es un programa moderno aunque crisimút derna pero hace lo mismo con las reaciones anti-modernas de finales de los sesenta proconiendo aś una síntesis a la que Ingersoll se ha referido cono "moderno critico". Aunque bien podrí denominarse post-moderno, un término que demasiado rápidamente se ha relacionado con la reación anti-moderna Una reaccín que, cómo ya se empieza a señalar desde alunos ámbitos

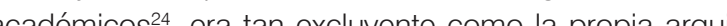
ectura que cilicaba si la inclusión es una de las cruerísticas fundamentales de la postmoderidad en arquectura el esfuerzo inclusivo que Koolhas prometíc sus teorís y que queda demostrado en edificios como el Congrexpo, convierte el proyecto de Lille en importante materializacín de su programa ideób́cico. Un proyecto donde las inevitables contradicciones que conleva a arquitectura de gran escala ayudan a entender mejor una verdadera post-modernidad, en la que ahora sí, lo uno lo otro son posibles simultáneamente.

22. Koolhaas, Rem: "Bigness, or the problem of large", op. cit. p. 495 .

23. Ingersoll, Richard: "Rem Koolhaas e l'ironia". En Casabella. № 610, Marzo 1994. pp. 16-20.

24. Reinhold Martin, coincidiendo con la visión de Charles Jencks señala que posiciones tan reaccionarias como las de Venturi fueron en el fondo modernas en un sentido estrictamente teórico, precisamente por su caracterer excllyente. Vease Martin, Reinhold: Utopia's 's Ghost, Architecture and Postmodernism, Again. (a) IT Press, 1998.

\section{Bibliografía}

Vittorio: The Possibility of an absolute architecture. Cambridge: The MIT Press, 2011

Balmond, Cecil: Informal. Munich - Berlín - Londres - Nueva York: Prestel, 2002

Ingersoll, Richard: "Rem Koolhaas e l'ironia". En Casabella. No 610. Marzo 1994. pp. 16-20.

Jencks, Charles: "Post-Modern Architecture"." En The lenguaje of Post-Modern Architecture. Londres: Academy Editions, 1977- Nueva York: Rizzol

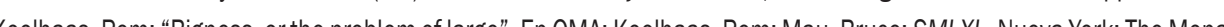

nacelli Press, 1995. pp. 494-517. Koolhas, Rem: "Whatever happened to Urbanism”" En OMA; Koolhaas, Rem; Mau, Bruce: SMLXL. Nueva York: The Monacelli Press, 1995. pp. $958-971$ Koolhaas, Rem; Zaera, Aejandro: "Encontrando Libertades: Conversaciones con Alejandro Zaera". En El Croquis. OMA/Rem Koolhaas, 1987-1993.

Koolhaas, Rem: Delirious New York: A Retroactive Manifesto for Manhattan. Nueva York: The Monacelli Press, 1978.

Martin, Reinhold: Utopia's 's Ghost, Architecture and Postmodernism, Again. Minesota: University of Minesota Press, 2010

Neumeyer, Fritz: “OMA's Berlin: The polemic island in the city". En Assamblage №. 11. April 1990. pp. 36-53.

OMA - AMO; Koolhaas, Rem: Content, Köln: Taschen, 2004.

Pozo y Barajas, Affonso del: La condición Postmoderna. Ideas de Ciudad. Sevilla: Secretariado de Publicaciones Universidad de Sevilla, Instituto .

Schriver, Lara: "OMA as a tribute to OMU: exploring resonances in the work of Koolhaas and Ungers". En The Journal of Architecture. Vol.13. № 3

Ungers, Oswald Mathias; Koolhaas, Rem; Riemann, Peter; Kolhoff, Hans; Ovaska, Arthur: "Cities within the City, Proposals by the Sommer Akademie

Venturi, Robert: Complexity and Contradicction in Architecture. Nueva York: The Museum of Modern Art, 1966.
Ignacio Senra Fernández-Miranda (Madrid, 1979), arquitecto por la ETSAM en 2005. Tras trabajar en el estudio de D. Rafael Moneo Valles, se graduo en 2009 en el Master of Science in Advance Architectural Design en la Graduate School of Architecture, Planning, and Preservation (GSAPP) de Columbia University, Nueva York, donde obtuvo el Premio Extraordinario Honor Award for Excellence in Design yel Premio Lucille S. Lowentifh Memorial Prize por su linvestigación sobre Urbanismo de alta densidad en Pekin. Actualmente desarrolla su Tesis Doctoral, un anâlisis critico sobre los libros Learning from las Vegas y Delirious New York 2011, ejerce como profesor ayudante en el Departamento de Proyectos Arquitectónicos de la ETSAM. 


\section{Autor imagen y fuente bibliográfica de procedencia}

Información facilitada por los autores de los artículos: página 17, 1 y 2 (Loghem, J. B. van: Bouwen / Bauen / Bâtir / Building Holland. Amsterdam: Kosmos 1932); página 18, 3 (Wiebenga archive (69-70), NAi, Rotterdam); página 20, 4 (Jan Molema), página 21, 5 (http://www.spotzi.com); página 22, 6 (Loghem, J. B. van: Bouwen / Bauen / Bâtir / Building Holland. Amsterdam: Kosmos 1932; Schütte-Lyhotski Archiv, Universität für angewannte Kunst, Vienna over a cadastral drawing on the internet: http://nah.cuzk.cz. Composed by Peter Bak and Jan Molema); página 23, 7 (Loghem, J. B. van: Bouwen / Bauen / Bâtir / Building Holland. Amsterdam: Kosmos 1932); página 24, 8 (Private collection Jan Molema), 9 (Jan Molema), 10 (Duiker Archive, NAi, Rotterdam); página 25, 11 (Van Loghem Bouwen / Bauen / Bâtir / Building Holland. Amsterdam: Kosmos 1932; C.A. Alberts and E.J. Jelles, Duiker 1890-1935, Forum, Amsterdam 1972); página 26, 12 ( Photo by courtesy of Arie den Dikken), 13 (Private collection Jan Molema); página 27, 14 ( Loghem, J. B. van: Bouwen / Bauen / Bâtir / Building Holland. Amsterdam: Kosmos 1932); página 28, 15 (Section from drawing in Duiker Archive, NAi Rotterdam); página 30, 16 (Multi-layer drawing on the basis of Duiker's site plan in Van Loghem Bouwen / Bauen / Bâtir / Building Holland. Amsterdam: Kosmos 1932. Composed by Jan Molema and Peter Bak); página 35, 1 y 2 (Francisco González de Canales); página 38, 3 y 4; página 40, 5; página 41, 6 (izda) (Koolhaas, Rem y Mau, Bruce: S, M, L, XL, Nueva York: The Monacelli Press, 1994), 6 (drcha) (Francisco González de Canales); página 41, 7; página 43, 8 y 9; página 44, 10 y 11; (Koolhaas, Rem y Mau, Bruce: S, M, L, XL, Nueva York: The Monacelli Press, 1994); página 49, 1 (Le Corbusier. En Boesiger, Willy. (Ed.): Le Corbusier Oeuvre complète. Volumen 1. 1910-29. 15a ed. Basilea: Birkhäuser Publishers - París: Fondation Le Corbusier, 1999. p. 189) ; página 51, 2 (Le Corbusier: Dibujo original del autor. FLC 10910. Fundación Le Corbusier. París, 1931), 3 (Le Corbusier: Fotografía del archivo de la FLC. FLC L2-4-41. Fundación Le Corbusier. París, s/f.); ; página 52, 4 (Le Corbusier. En Boesiger, Willy. (Ed.): Le Corbusier Oeuvre complète. Volumen 4. 1938-4. $11^{\mathrm{a}}$ ed. Basilea: Birkhäuser Publishers - París: Fondation Le Corbusier, 1999. p. 139) ; página 53, 5 (Le Corbusier: Dibujo original del autor. FLC 19238. Fundación Le Corbusier. París, 1936); página 54, 6 (Le Corbusier. EnBoesiger, Willy. (Ed.): Le Corbusier Oeuvre complète. Volumen 5. 1946-52. 11ª ed. Basilea: Birkhäuser Publishers París: Fondation Le Corbusier, 1999. p. 37) ; página 55, 7 (Le Corbusier: Dibujo original del autor. FLC 32294. Fundación Le Corbusier. París, 1951), 8 (Le Corbusier: Dibujo original del autor. FLC 2892. Fundación Le Corbusier. París, 1951), 9 (Le Corbusier. En Boesiger, Willy. (Ed.): Le Corbusier Oeuvre complète. Volumen 5. 1946-52. 11ª ed. Basilea: Birkhäuser Publishers - París: Fondation Le Corbusier, 1999. p. 121) ; página 56, 10 (Le Corbusier. En Petit, Jean. (Ed.): Un couvent de Le Corbusier. París: Éditions de Minuit, 1961. p. 112); página 58, 11 (Le Corbusier. EnBoesiger, Willy. (Ed.): Le Corbusier Oeuvre complète. Volumen 7. 1957-65. $7^{a}$ ed. Basilea: Birkhäuser Publishers - París: Fondation Le Corbusier, 1999. p. 33), 12 (Le Corbusier: Dibujo original del autor. FLC 31197. Fundación Le Corbusier. París, 1960), 13 (Le Corbusier: Dibujo original del autor. FLC 11644. Fundación Le Corbusier. París, 1963); página 60, 14 (Le Corbusier: Dibujo original del autor. FLC 28450. Fundación Le Corbusier. París, 1963), 15 (Le Corbusier: Dibujo original del autor. FLC 28460. Fundación Le Corbusier. París, 1963), 16 (Le Corbusier: A propósito del urbanismo. Barcelona: Editorial Poseidón, 1980. p. 144); página 63, 1 (From the archive of The Metropolitan Museum of Art, New York); página 64,2 (Courtesy of the digital archive of architectural images archINFORM), 3 y página 66, 4 (Wolfe, Ross: The Charnel-House. [en línea] New York. Disponible en www.thecharnelhouse.org); página 68, 5 (From the archive of the DEUTSCHES ARCHITEKTURMUSEUM), 6 (composed image, using different PROUNS of Lissintzky from www.wikipaintings.org); página 70, 7 (Composed image, using two drawing from the digital archive of architectural images archINFORM), 8 (From the permanent Collection of the Museo Nacional Centro de Arte Reina Sofía); página 74, 9 (Render of the project CAl Periféricos of the Empresa de Desarrollo Urbano de Medellín, EDU); página 78, 1 (El Croquis n.53, OMA/Rem Koolhaas, 1987-1993. Madrid: editorial El croquis, 1992, p. 167), 2 (Faucherau, Serge: Malévich, Barcelona: Ediciones Polígrafa, 1992, p. 143, figura 37), 3 (Aureli, Pier Vittorio: The Possibility of an absolute architecture. Cambridge: The MIT Press, 2011, p. 179); página 81, 4 (Gargiani, Roberto: Rem Koolhaas / OMA, the Construction of Maravilles. Lausanne: EPFL Press, 2008, p. 26 ), 5 (El Croquis n.53, OMA/Rem Koolhaas, 19871993. Madrid: editorial El croquis 1992, p. 169); página 82, 6 (El Croquis n.53, OMA/Rem Koolhaas, 1987-1993. Madrid: editorial El croquis 1992, p. 178), 7 (El Croquis n.53, OMA/Rem Koolhaas, 1987-1993. Madrid: editorial El croquis 1992, p. 173), 8 (El Croquis n.53, OMA/Rem Koolhaas, 1987-1993. Madrid: editorial El croquis 1992, p. 85), 9 (Aureli, Pier Vittorio: The Possibility of an absolute architecture. Cambridge: The MIT Press, 2011, p. 207); página 84, 10 (Venturi, Robert: Complexity and Contradicction in Architecture. New York: The Museum of Modern Art, 1966. p. 74), 11 ( AV Monografías 51-52, Enero-Abril 1995, p. 172), 12 (Arquitectura Viva n. 39, Noviembre-Diciembre 1994, p.43); página 86, 13 y 14 (OMA; Koolhaas, Rem; Mau, Bruce: SMLXL. Nueva York: The Monacelli Press, 1995. pp. 810-811); página 90, 1 (Courtesy of The MIT Press, from Grant Hildebrand, Designing for Industry: The Architecture of Albert Kahn. p.46), 2 (From the Collections of The Henry Ford); página 92, 3 (Nelson, George: Industrial Architecture of Albert Kahn, Inc. New York: Architectural Book Publishing Company, Inc, 1939, p.85. Foto: Hedrich-Blessing); página 93, 4 (Goldsmith, Myron: The Tall Building: the Effects of Scale, IIT, Chicago, 1953 (Tesis doctoral no publicada, revisada en. 1977 y 1986). [Versión consultada en: Goldmith, Myron y Werner Blaser (ed), Buildings and Concepts. New York: Rizzoli International Publications, 1987. pp.8-22.], p. 15), 5 (Goldsmith, M., op. cit. p. 17); página 94, 6 y página 95, 7 (Silvia Colmenares Vilata); página 96, 8 (Achilles, Rolf; Kevin Harrington and Charlotte Myhrum (ed.) Mies van der Rohe, architect as educator. Catalogue for the exhibition, 6 June through 12 July 1986 . Mies van der Rohe Centennial Project, IIT. Chicago: University of Chicago Press, 1986. pp.126 // Nelson, G. Op. cit. p. 38); página 97, 9 (Carter, Peter: Mies van der Rohe at Work. New York: Praeger, 1974. [Ed. Consultada: London: Phaidon, 1999, p. 8); página 98, 10 (Silvia Colmenares Vilata); página 99, 11 (Hvattum, Mari; Hermansen Cordua, Christian (eds.) Tracing Modernity: Manifestations of the Modern in Architecture and the City. London: Routledge, 2004. p. 126), 12 (Wesemael, Pieter van. Architecture of Instruction and Delight: A Socio-Historical Analysis of World Exhibitions As a Didactic Phenomenon (1798-1851-1970).Rotterdam: Uitgeverij 010, 2001. p. 170. (Manipulada)); página 100, 13 (Cedric Price fonds. Collection Centre Canadien d'Architecture/ Canadian Centre for Architecture, Montréal), 14 (Cedric Price fonds. Collection Centre Canadien d'Architecture/ Canadian Centre for Architecture, Montréal); página 101, 15 (Silvia Colmenares Vilata); página 106, 1, 2 y 3; página 108, 4 y 5 (Luis Palacios Labrador, 2011); página 110, 6 (Dibujo Luis Palacios Labrador, 2011. Fotografía: Strauven, Francis: Aldo Van Eyck. The Shape of Relativity. Amsterdam: Architectura \& Natura, 1998, pp. 375); página 113, 7 (Strauven, Francis: Aldo Van Eyck. The Shape of Relativity. Amsterdam: Architectura \& Natura, 1998, pp. 378), 8 (Strauven, Francis: Aldo Van Eyck. The Shape of Relativity. Amsterdam: Architectura \& Natura, 1998, p. 402); página 114, 9 (Risselada, Max; Van den Heuvel, Dirk: Team 10, 1953-81, in search of a Utopia of the present. Rotterdam: Nai Publishers, 2005, p. 117), 10 (Aldo van Eyck. The Web and the Labyrinth. En Lotus International. V.11. 1976); página 115, 11 (Sarkis, Hashim: Case: Le Corbusier's Venice Hospital and the Mat Building Revival. Munich: Prestel Verlag, 2001, p. 41); página 120, 1 (Antonio Millán Gómez, Marisol Jiménez, Julio Alan Latre y Víctor Díaz-Asensio García); página 123, 2 (Latre Cabrera, Julio Alan y Jiménez Rivera, Marisol); página 124, 3 (3.a. Busquets, Joan: El centro Histórico de Barcelona, un Pasado con futuro. Barcelona: Ajuntament de Barcelona, Foment Ciutat Vella; Universitat Politècnica de Catalunya, 2003; 3.b www.europeana.eu/portal/record/91932/CA529705B53B599FDD3C9A1ED7D5130497F5D000.html), 4 (Antonio Millán Gómez, Marisol Jiménez, Julio Alan Latre y Víctor Díaz-Asensio García); página 126, 5 (Latre Cabrera, Julio Alan y Jiménez Rivera, Marisol); página 128, 6 (llustre Municipalidad de Santiago); página 129, 7 (Archivo Visual de Santiago (www.archivovisual.cl) e llustre Municipalidad de Santiago / Santiago Centro. pp. 24-25); página 130, 8 (Lazo Mella, Felipe ; Millán Gómez, Antonio); página 131, 9 (Atlas del Proyecto de saneamiento del subsuelo de BarceIona. Lamina 3. Archivo Histórico de la Ciudad de Barcelona) 